\title{
The importance of the recovery treatment in increasing the quality of the lives of the patients with the carpal tunnel syndrome
}

\author{
Sinziana Calina Silisteanu ${ }^{1}$, Elisabeta Antonescu ${ }^{2}$, Valerica Moisii ${ }^{3}$ \\ ${ }^{1}$ Railway Hospital Iasi - Specialty Ambulatory of Suceava - "Stefan cel Mare" University of \\ Suceava \\ 2 Physicist - "Lucian Blaga” University of Sibiu \\ ${ }^{3}$ Physical therapist
}

\begin{abstract}
The hand is a very important segment in gestures, functionality and the achievement of ADL. The Carpal tunnel syndrome was described for the first time by Paget in 1854 , being a very frequent health condition of the extremity in the upper limb caused by the compression of the median nerve at the level of the fibre bone tunnel at the level of the hand wrist. The incidence of the Carpal tunnel syndrome is approximately $0.125 \%-1 \%$ /year, having a prevalence of $5-15 \%$. Among the patients diagnosed with the Carpal tunnel syndrome, $80 \%$ are over 40 years old, the women being more affected than the men. The Carpal tunnel syndrome is a disability issue with social and economic consequences, being a burden for the society. The recovery is conservative and it consists of immobilisation by orthesis at the hand level, crio-therapy, kinetic therapy, electric therapy (laser, ultrasound, low and average frequency electrical power) or by surgical intervention. This study tries to point out if it is possible to influence the quality of life and the individuals' level of functional independence by applying therapeutic protocols specific to this health condition. The patients diagnosed with the Carpal tunnel syndrome received a complex treatment which included electric therapy (of low and average frequency, TENS, laser and ultrasound), massage for the upper limb and kinetic therapy, with a length of 15 days, then the patients were called for the checkup 30 days later. It is important to set clear objectives for the recovery programmes and to create optimised treatment schemes. They confirm the hypothesis based on which the research was conducted. Therefore, any health condition for this segment may influence the quality of patients' life, with involvement in the achievement of the daily activities and the social professional insertion.
\end{abstract}

Key words: the Carpal tunnel syndrome, disability, the quality of life, study 


\section{Introduction.}

The Carpal tunnel syndrome was described for the first time by Paget in 1854, being a very frequent health condition of the extremity in the upper limb caused by the compression of the median nerve at the level of the fibre bone tunnel at the level of the hand wrist. From an anatomic point of view, the Carpal tunnel is an inextensible formation being in bone touch in three parts (the Carpal bones) and a ligament touch (the transverse Carpal ligament) [1]. The dimensions of the Carpal canal may be reduced even to a simple flexion of the hand at $90^{\circ}$.

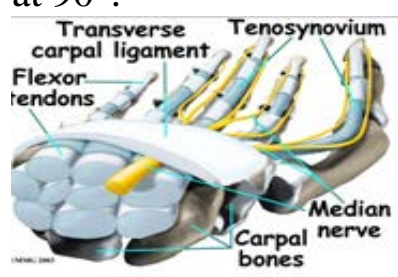

Figure no. 1 The hand anatomy

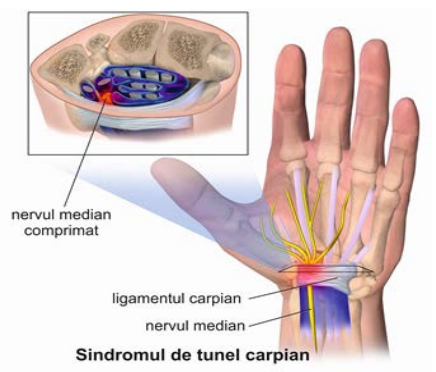

Figure no. 2 The structure of the Carpal canal

The median nerve is located under the transverse Carpal ligament and it has the role to innervate sensitively the palmar face, the ternary eminence, especially the polix, the index, the middle finger and half of the ring finger.

There are nine flexor tendons in the Carpal canal that allow the fingers to move in the flexion. These tendons are covered by a synovial membrane that allows the glide for the contraction/relaxation movement.

The incidence of the Carpal tunnel syndrome is approximately $0.125 \%-1 \%$ /year, having a prevalence of 5-15\% [2].

Among the patients diagnosed with the Carpal tunnel syndrome, $80 \%$ are over 40 years old, the women being more affected than the men $(5: 1)$. The health condition is located bilaterally but the dominating hand is the first one affected and the evolution is severe .

Among the factors that caused it, the different studies mentioned:endocrinological and rheumatic diseases, infections, the median artery thrombosis, fibrotic inflammatory modifications of the synovial, bone, muscular and neurovascular anomalies, tumors, traumatic lesions, pregnancy, correlated especially with the daily gestures and with the manual activities.

Some of the most exposed persons to this health condition are the ones who work on the computer, the dentists but also the ones who make sudden repetitive movements of hand flexion/extension. There are several important factors that cause this health condition: the force with which the movement is made, the temperature of the environment, the performance posture and the alignment of the elements that make up the fist and the hand.

From the etiological point of view, the Carpal tunnel syndrome may have an idiopathic cause . The Carpal tunnel syndrome is a disability issue with social and economic consequences, being a burden for the society

The Carpal tunnel syndrome is generally treated by surgical intervention . For example, there were approximately 
300,000 interventions performed in Germany annually, out of which almost 90\% were treated in the ambulatory . $78-80 \%$ of the persons with the Carpal tunnel syndrome who were solved by surgery returned to their professional activities.

Among the incriminated factors for the increase of the absence length in the professional activity of the persons with the Carpal tunnel syndrome, we mention the female gender, professional activities (repetitive manners, the performance of movements at the fist level with the increased force and endurance), pregnancy , age and issues of nervous conduct that existed before the surgery .

There are studies that did not point out the connection among age, gender and the professional activity but the women complained of more painful symptoms before the surgery [3].

Even though the elderly had more severe forms of the Carpal tunnel syndrome, they did not have intense symptomatology [4] but they were less satisfied after the surgical intervention.

The symptoms are motion restriction, pain, paraesthesias at the level of the hand and of the forearm. The recovery is conservative and it consists of immobilisation by orthesis at the hand level, crio-therapy, kinetic therapy, electric therapy (laser, ultrasound, low and average frequency electrical power) or by surgical intervention.

There are studies which show that the recovery of the patients with the Carpal tunnel syndrome is different according to the moment when the health condition was noticed, the surgical intervention, the recovery methods and the patient's compliance. There are many patients who consider that the surgical intervention may improve the symptoms or it may even make them disappear. This is possible only in the acute stages of the Carpal tunnel syndrome and at the beginning of the health condition. As well, the surgery is optimal for the situation when the symptoms return repeteadly, the pain sensation increases and the joint motion is limited, leading to the decrease of the flexion/extension angle and the development of ADL. Approximately $75-90 \%$ of the persons who underwent a surgical intervention continue to show painful symptoms and paraesthesias [5]. There are research studies which show that the surgical treatment seems to be more efficient than the applied orthesis and the administered AINS.

A study conducted in 2014 shows that the diagnosis of the Carpal tunnel syndrome by using the muscular skeletal ultrasonography as well as the electronic myography are less sensitive in comparison to the local physical examination [6].

The clinical diagnosis relies especially on the physical examination and on the following tests: TINEL, PHALEN and DURKAN. In the Carpal tunnel syndrome, the flexion position of the fist compresses the median nerve more than it was compressed in the neutral position. The DURKAN test consists of applying pressure in the Carpal region, with the polix of both hands for 30 seconds, which triggers the symptomatology characteristic for the Carpal tunnel syndrome.

The speed of conducing the median was measured by neurophysiological methods. The diagnosis of the Carpal tunnel syndrome may be put by high resolution ultrasonography [7], physical examination and electronic myography [8].

The anatomic evaluation of the Carpal tunnel is very important for the diagnosis and for the treatment. The mechanical stress may determine the deformation of the myelin sheath [9] at the same time with the occurrence of morphological ischemic modifications accompanied by paraesthesias, frequently at 
night at the flexion movement of the hand [10].

While setting the diagnosis of the Carpal tunnel syndrome, it is important to have in view the imagery techniques, the ultrasonography and the IRM [11] which can confirm the oedema lesions at the median level at the entrance into the Carpal tunnel but also its flattening and the modifications of the flexor retinaculum at the exit of the tunnel [12].

The ultrasonography is used for the evaluation of the median nerve. There are studies that introduce an appreciation of the median nerve's dimensions in 8 points located on the cross-section area of the median nerve and all along the Carpal canal [13].

The studies that analyse the nervous conduct and imagery by ultrasonography may offer supplementary anatomic information related to the pathological modifications of the median nerve [14]. The translation of the median nerve during the movements of the fingers was analysed by ultrasonography. Thus it was found out that the median nerve glides transversally during the movements of the hand and of the fingers, and namely: the median nerve of a healthy person moves more than the median nerve of a person with the Carpal tunnel syndrome.

The test estimated quantitatively the dysfunction of the median for the patients diagnosed with the Carpal tunnel syndrome and subsequently it was confirmed by IRM and neurography, which could prove these previous back movements of the median in the Carpal canal . The imagery allowed the appreciation of the transversal cross-section area of the median and the modifications of the thickness in the flexor retinaculum, with a sensitivity of approximately $90 \%$ and a specificity of approximately $85 \%$.

Having in view that the median nerve has a flexible and mobile structure, it can be compressed and relaxed at the same time as the movements of the flexor tendons , it is pulled or on the contrary "crushed" by the flexor tendons towards the Carpal transversal ligament. During the movements of the hand and of the fingers, the transversal glide but also the longitudinal excursion of the median was ampler for healthy persons in comparison to the ones diagnosed with the Carpal tunnel syndrome. The movement of the median nerve depends on the anatomic vascular characteristics amd the aspect of the other flexor tendons.

The specialty literature presented the fact that the active movements of the fingers may affect the movement of the median in the palm-dorsal direction or in the radialulnar one [15]. Initially the median nerve has a neutre position. During the total flexion of the fist, the median nerve glides first transversally to the ulna and then at the extension of the fingers it turns towards the radial.

The researchers pursued the changes made in the dynamics by the median nerve and by the flexor tendons, by evaluating the cinematics in the Carpal tunnel and by trying to mention the pathogeny of the Carpal tunnel syndrome [16], [17]. The superficial flexor tendon of the third finger is located near the median whereas behind and further from the latter are located the flexor tendons of the index and the long flexor of the polix. At the flexion movement of the fingers, the median and the flexor tendon of the index approaches whereas, at the extension movement of the fingers, the median and the flexor tendon of the index are further.

\section{Purpose}

This study tries to point out if it is possible to influence the quality of life and the individuals' level of functional independence by applying therapeutic protocols specific to this health condition. 
Are there modalities that can restrict the flexion and extension movement at the hand level, that can prevent the inflammation and the pressure from increasing inside the Carpal tunnel?

\section{Material and method.}

The study was conducted on a group of 15 patients diagnosed with the Carpal tunnel syndrome, most of them after surgery. The inclusion criteria were: the presence of a pathology that can determine the compression of the median nerve, the presence of: spontaneous pains, paraesthesia and paresis accompanied by functional impotency. The exclusion criteria were: tumors, vascular necrosis, bone pathologies, cervical hernia, multiple sclerosis and other neurological diseases.

The evaluation of the patients was made by using scales, namely: for pains the visual analogous scale (VAS), for the functionality of the hand necessary to achieve the ADL - Jebsen-Taylor test (JBT), for the appreciation of the quality of life the scale QOL.

The objectives of the recovery programme were: to keep the tonicity of the muscular tissue, to avoid the ligamentary retractions to preserve the segmentary joint function, to increase the muscular force and endurance, to reintegrate the patients from a social and professional point of view.

The patients diagnosed with the Carpal tunnel syndrome received a complex treatment which included electric therapy (of low and average frequency, TENS, laser and ultrasound), massage for the upper limb and kinetic therapy, with a length of 15 days, then the patients were called for the checkup 30 days later.

We used the correct posture, the orthesis, the passive mobilisations, the techniques of neural proprioceptive facilitation, ergo-therapy, aiming at achieving ability, balance and coordination.
The group included 20 patients out of which 12 of female gender and 8 of male gender.

The pain score may be represented graphically from a statistical point of view in the three evaluation stages: initial, final and control.

The Jebsen-Taylor test allows evaluating the functions of the useful hand in order to achieve the ADL. The test was applied to the dominating limb (MD) and to the non-dominating one (ND).

\section{Results}

Table no.1 Evolution of the pain score

\begin{tabular}{|l|c|c|c|}
\hline & VAS initial & VAS final & VAS control \\
\hline Average & 6.6 & 4.3 & 2.6 \\
\hline Median & 6.5 & 4 & 2 \\
\hline Standard deviation & 0.966092 & 1.05935 & 0.843274 \\
\hline Student's t test & 0.030585 & 0.050112 & \\
\hline
\end{tabular}

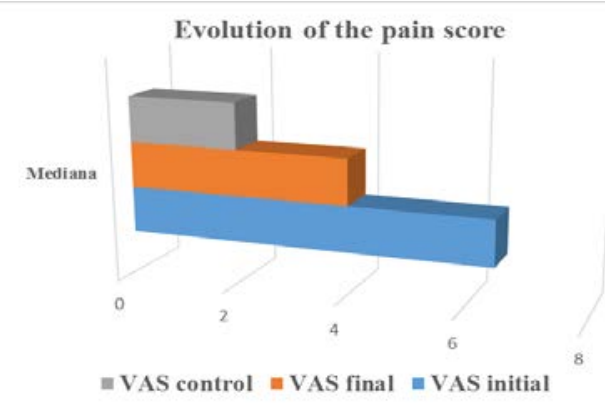

Figure no.3 Evolution of the pain score

One of the tests is the return of the playing card whereas the evaluation is made at the beginning and at the end of the recovery treatment.

Table no. 2 Analysis of the item evolution "Return of playing cards"

\begin{tabular}{|c|c|c|c|c|}
\hline Statistical & Return of & Return of & Return of & Return of \\
\hline \multirow[t]{2}{*}{ elements } & playing cards & playing cards & playing cards & playing cards \\
\hline & (initial) MD & (final) MD & (initial) MD & (final) MND \\
\hline Average & 6.242 & 4.996 & 6.917 & 5.029 \\
\hline Median & 6.242 & 4.996 & 6.99 & 5.01 \\
\hline Standard deviation & 0.6615 & 0.71 & 0.4874 & 0.4834 \\
\hline Student's t-test & 0.0011 & & 0.0143 & \\
\hline
\end{tabular}




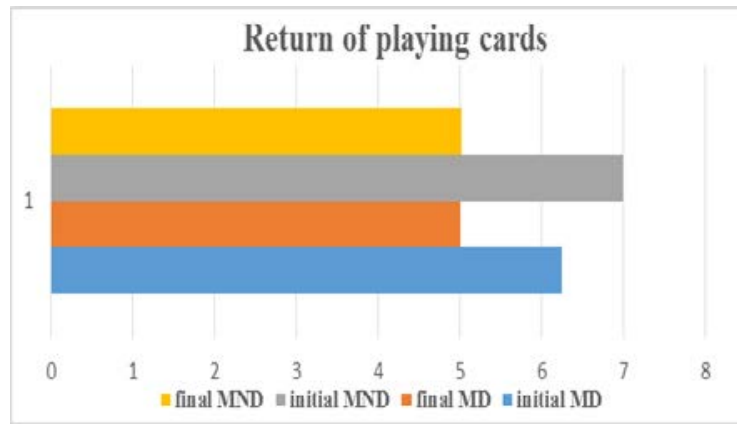

Figure no. 4 Representation of item "Return of playing cards"

Another test is represented by the simulation of the eating activity whereas the evaluation is made for the dominating limb and for the non-dominating limb, at the beginning and at the end of the recovery treatment.

Table no. 3. Analysis of the item evolution "Eating simulation"

\begin{tabular}{|l|l|l|l|l|}
\hline Statistical & Eating & Eating & Eating \\
elements & $\begin{array}{l}\text { simulation } \\
\text { (initial) MD }\end{array}$ & $\begin{array}{l}\text { simulation } \\
\text { (final) MD }\end{array}$ & $\begin{array}{l}\text { Eating } \\
\text { (initial) MND } \\
\text { simulation } \\
\text { (final) MND }\end{array}$ \\
\hline Average & 7.72 & 96.194 & 9.047 & 7.863 \\
\hline Mediana & 7.84 & 6.18 & 9.105 & 8.005 \\
\hline Standard deviation & 0.28639 & 0.228969 & 0.279923 & 0.343481 \\
\hline Student's t-test & 0.03592 & & 0.0026 & \\
\hline
\end{tabular}

Eating simulation

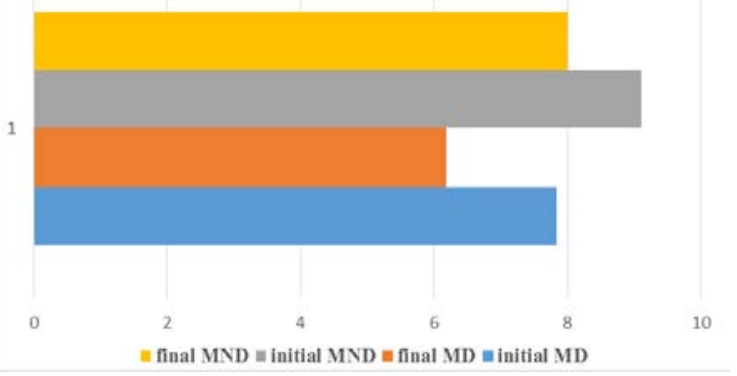

Figure no. 5 Representation of the item "Eating simulation"

The third test is represented by the ability to move small common objects for the dominating limb and for the nondominating one whereas the evaluation is made at the beginning and at the end of the recovery treatment.

Table no. 4 Analysis of the item evolution "Moving a small object"

\begin{tabular}{|c|c|c|c|c|}
\hline Statistical & Moving a & Moving a & Moving a & Moving a \\
\hline \multirow[t]{2}{*}{ elements } & small object & s small object & small object & small object \\
\hline & initial MD & final MD & initial MND & final MND \\
\hline \begin{tabular}{|l|} 
Average \\
\end{tabular} & 7.973 & 6.031 & 7.595 & 6.165 \\
\hline \begin{tabular}{|l|} 
Median \\
\end{tabular} & 8.025 & 6.2 & 7.61 & 6.085 \\
\hline Standard deviation & 0.441363 & 0.510696 & 0.365065 & 0.374381 \\
\hline Student's t-test & 0,00131680 & & 0.0059242 & \\
\hline
\end{tabular}

The fourth test was moving full big bottles whereas the evaluation is made at the beginning and at the end of the recovery treatment for the dominating limb and for the non-dominating one.

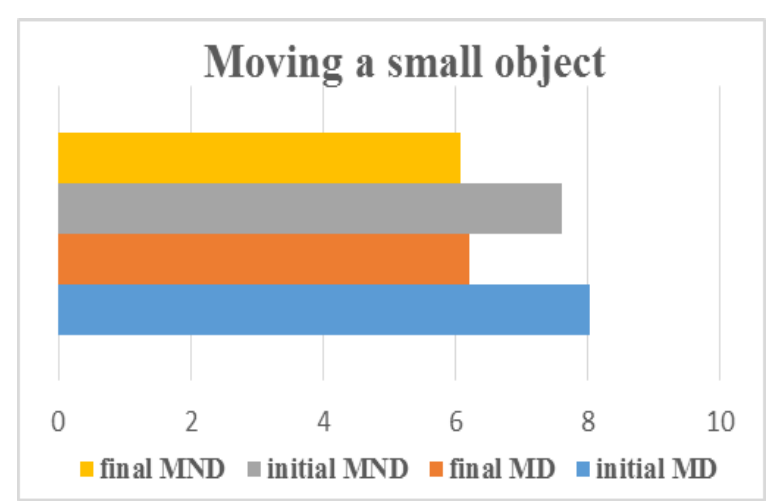

Figure no. 6 Representation of the item "Moving a small object"

Table no. 5 Analysis of the item evolution "Moving full big bottles"

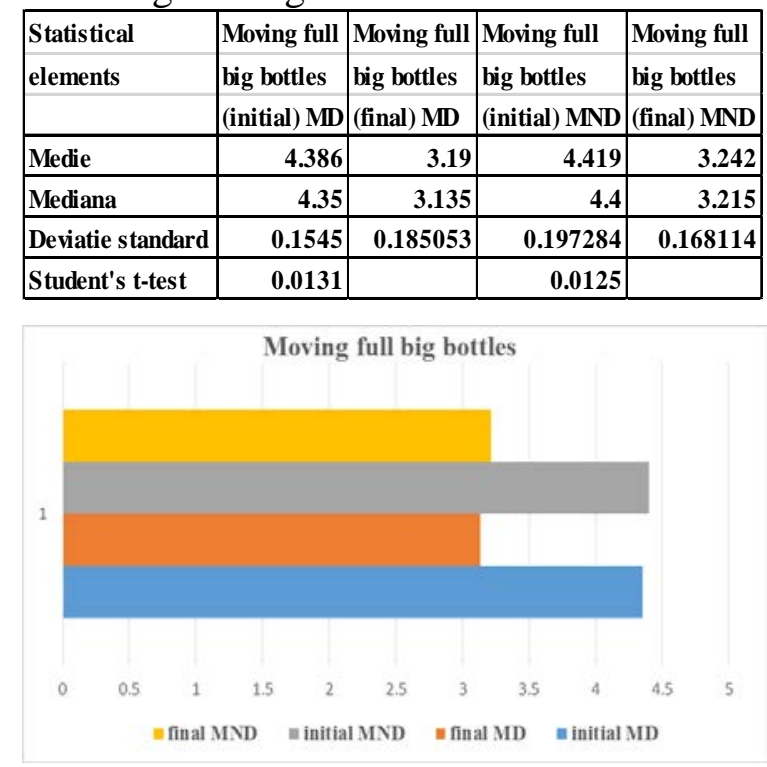

Figure no. 7 Representation of the item "Moving full big bottles" 
The quality of life for the patients diagnosed with the Carpal tunnel syndrome was achieved with the help of the QOL scale for the initial, final and control moment. As for the analysis of the modifications in the questionnaire that evaluates the quality of life for the patients included in the study, there are improvements of less than 10 points between the initial stage and the final one.

Table no. 6 Analysis of evolution QLS

\begin{tabular}{|l|r|r|r|}
\hline Statistical & & & \\
elements & QLS initial & QLS final & QLS control \\
\hline Average & 78.533 & 83266 & $\mathbf{8 8 . 8 6 6}$ \\
\hline Mediana & 78 & 83 & 89 \\
\hline Standard deviation & 6.9781 & 5.1473 & 3.6029 \\
\hline Student's t-test & 0.00063 & 0.00071 & 0.0014 \\
\hline
\end{tabular}

Analysis of evolution QLS

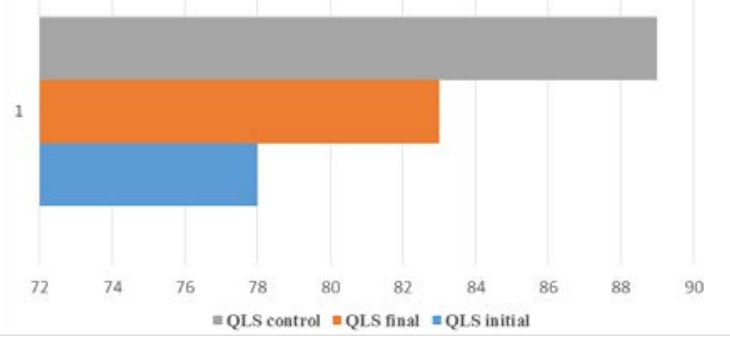

Figure no. 8 Representation of evolution QLS

\section{Conclusions}

Even though the patients diagnosed with the Carpal tunnel syndrome had the dominant limb affected in a proportion of $93 \%$ the evaluation tests were applied for the dominating limb and for the non-dominating one.

It was found that especially girls and women had the Carpal tunnel syndrome, the pathology was varied and the age of diagnosing the health condition did not matter.
It is important to set clear objectives for the recovery programmes and to create optimised treatment schemes. They confirm the hypothesis based on which the research was conducted. The hand is a very important segment in gestures, functionality and the achievement of ADL. Therefore, any health condition for this segment may influence the quality of patients' life, with involvement in the achievement of the daily activities and the social professional insertion.

The limits of the study were represented by the small number of patients who agreed with this study, the impossibility to monitor them at home, the patients' subjectivity but also the existence of other comorbidities that influenced the development of the recovery activity.

\section{References}

1.Tai-Tzung Kuo, Ming-Ru Lee,Yin-Yin Liao, Jiann-Perng Chen, Yen-Wei Hsu, and Chih-Kuang Yeh, Assessment of Median Nerve Mobility by Ultrasound Dynamic Imaging for Diagnosing Carpal Tunnel Syndrome, PLoS One. 2016; 11(1): e0147051.Published online 2016 Jan 14. doi: 10.1371/journal.pone.0147051

2. Cartwright M.S., Hobson-Webb L.D., Boon A.J., Alter K.E., Hunt C.H., Flores V.H. Evidence-based guideline: neuromuscular ultrasound for the diagnosis of carpal tunnel syndrome. Muscle Nerve. 2012;46(2):287-293.

3.Ibrahim T, Majid I, Clarke M, Kershaw CJ. Outcome of carpal tunnel decompression: the influence of age, gender, and occupation. Int Orthop. 2009;33:13051309.

4.Povlsen B, Aggelakis K, Koutroumanidis M. Effect of age on subjective complaints and objective severity of carpal tunnel syndrome: prospective study. JRSM Short Rep. 2010;1:62. 
5.Disable Glossary Links, Informed Health Online [Internet], Carpal tunnel syndrome: When is surgery an option and when is it necessary?, Created: November 5, 2014; Next update: 2017.

6. Arnaldo Gonçalves de Jesus Filho AG, do Nascimento BF, Amorim Mde C, Naus RA, Loures Ede A, Comparative study between physical examination, electroneuromyography and ultrasonography in diagnosing carpal tunnel syndrome, Rev BrasOrtop.2014Sep 16;49(5):446-51

7.Kang S., Kwon H.K., Kim K.H., Yun H.S. Ultrasonography of median nerve and electrophysiologic severity in carpal tunnel syndrome. Ann Rehabil Med. 2012;36(1):72-79.

8.Rahmani M., Ghasemi Esfe A.R., VaziriBozorg S.M., Mazloumi M., Khalilzadeh O., Kahnouji H. The ultrasonographic correlates of carpal tunnel syndrome in patients with normal electrodiagnostic tests. Radiol Med. 2011;116(3):489-496.

9.Fowler J.R., Maltenfort M.G., Ilyas A.M. Ultrasound as a first-line test in the diagnosis of carpal tunnel syndrome: a costeffectiveness analysis. Clin Orthop Relat Res.2013;471(3):932-937.

10.De-la-Llave-Rincon A.I., Laguarta-Val S., Arroyo-Morales M., Martinez-Perez A., Pareja J.A., Fernandez-de-Las-Penas C. Characterization of pain in patients with carpal tunnel syndrome according to electromyographic severity criteria. Rev Neurol.2012;54(7):407-414.

11.Wilson D., Allen G.M. Imaging of the carpal tunnel. Semin Musculoskelet Radiol.2012; 16(2):137-145.

12.Chan K.Y., George J., Goh K.J., Ahmad T.S. Ultrasonography in the evaluation of carpal tunnel syndrome: diagnosis criteria and comparison with nerve conduction studies.Neurology Asia. 2011;16(1):57-64 13.Shu-Fang Chen, Cheng-Hsien Lu, ChiRen Huang,Yao-Chung Chuang,Nai-Wen Tsai,Chiung-Chih Chang,Wen-Neng Chang,
Ultrasonographic median nerve crosssection areas measured by 8-point "inching test" for idiopathic carpal tunnel syndrome: a correlation of nerve conduction study severity and duration of clinical symptoms, BMC Med Imaging. 2011; 11: 22.Published online 2011 Dec 21

14.Kuo TT, Lee MR, Liao YY, Chen JP, Hsu YW, Yeh CK, Assessment of Median Nerve Mobility by Ultrasound Dynamic Imaging for Diagnosing Carpal Tunnel Syndrome, PLoS One. 2016 Jan 14;11(1):e0147051.

15.Korstanje JW, Scheltens-De Boer M, Blok JH, Amadio PC, Hovius SE, Stam HJ, et al. Ultrasonographic assessment of longitudinal median nerve and hand flexor tendon dynamics in carpal tunnel syndrome. Muscle Nerve. 2012. May;45(5):721-9. 16.Liao YY, Lee WN, Lee MR, Chen WS, Chiou HJ, Kuo TT, et al. Carpal tunnel syndrome: US strain imaging for diagnosis. Radiology. 2015. April;275(1):205-14. 17.van Doesburg MH, Henderson J, Mink van der Molen AB, An KN, Amadio PC.Transverse plane tendon and median nerve motion in the carpal tunnel: ultrasound comparison of carpal tunnel syndrome patients and healthy volunteers. PLoS One. 2012;7(5):e37081 